\title{
Glossopharyngeal breathing can allow a lung expansion greater than inspiratory capacity in muscular dystrophy
}

\author{
To the Editor:
}

Glossopharyngeal breathing (GPB), also called "frog breathing", is a positive pressure breathing technique that uses muscles of the mouth and pharynx to propel small volumes of air ("gulps") into the lungs. It consists of three phases: 1) mouth opening so that air enters the oral and pharyngeal cavities; 2) mouth closure with tongue and pharyngeal muscles propelling a gulp of air through the open glottis raising intrathoracic pressure; 3 ) glottis closure to ensure the air trapped into the lungs $[1,2]$. These three phases are repeated several times until a comfortable lung expansion is reached. GPB is known to be able to increase lung volumes above physiological total lung capacity (TLC), therefore increasing vital capacity [2-5]. This is a consequence of an increase in lung volume, but also of intrapulmonary gas compression. The physiological factors limiting TLC are the effect of the inspiratory muscles, which rapidly decreases, and the elastic expiratory (inward) recoil of lungs and chest wall, which increases. By contrast, TLC during GPB seems to be limited by sensation rather than by respiratory muscles and/or the mechanics of the respiratory system [3]. GPB is largely used by divers because it increases the amount of oxygen/carbon dioxide stored in the body, therefore prolonging their breath-hold duration $[3,6,7]$. GPB can also be used as lung inflation therapy to assist the ventilatory pump when it becomes weak or impaired, as in the case of cervical spinal cord injury and/or neuromuscular diseases [8].

Muscular dystrophy (MD) is a group of inherited myopathies characterised, for example, by progressive skeletal muscle wasting and weakness, including of the respiratory muscles, leading to ventilatory pump failure. In addition, the lung and the chest wall become less distensible, increasing the load against which the ventilator pump must contract. With the progression of $\mathrm{MD}$, a progressive imbalance between the load to be overcome and the capacity to overcome it occurs [9]. Regular mobilisation is important to prevent chest wall contractures and/or lung restriction. These patients, therefore, may benefit from GPB as they can recruit the lung, maintain lung and/or chest wall compliance, sustain ventilation for several hours, diminish atelectasis, and improve voice and cough efficacy $[2,5,10]$. GPB sometimes is learned by the patient of their own accord or the patient can be trained by specialist physiotherapists. The positive effects of GPB in patients with Duchenne muscular dystrophy (DMD) have been reported by three groups in terms of increased peak cough flow [10] and expiratory volume [2, 5].

Measurements during GPB are sometimes difficult if using a mouthpiece and pneumotachometer, particularly in patients with macroglossia or sever facial muscle weakness. Lung and chest wall volume variations can alternatively be evaluated by external measurements of chest wall surface motion, like opto-electronic plethysmography (OEP). OEP uses infrared cameras and passive reflective markers on the chest wall, thus leaving the mouth free. This system also provides information on the contribution of the ribcage and the abdomen to breathing [11-13].

We used an eight TV camera OEP system to measure chest wall and thoraco-abdominal volume variations during quite breathing, inspiratory capacity (IC) and self-learned GPB in one DMD, one Becker muscular dystrophy (BMD) patient and a competitive breath-hold diver (healthy control; HC) in two postures.

@ERSpublications

In muscular dystrophy, glossopharyngeal breathing is effective in presence of severe dysphagia and diaphragmatic weakness: it recruits the lung above IC, reversing paradoxical respiratory abdominal motion with a presumably more homogeneously distributed pulmonary ventilation http://bit.ly/ 2LyXDEL

Cite this article as: LoMauro A, Banfi P, D’Angelo MG, et al. Glossopharyngeal breathing can allow a lung expansion greater than inspiratory capacity in muscular dystrophy. Eur Respir J 2019; 54: 1801938 [https://doi.org/10.1183/13993003.01938-2018]. 
The supine position was performed on a rigid support and a 52 marker model was used. In the sitting position, the DMD patient was monitored while seated on his wheelchair using the same 52 marker model, whereas an 89 marker model was used for the BMD patient and HC who were seated on the bed without support. All the subjects were asked to reach total lung capacity and then to start GPB up to the maximum volume. They were free to control the timing and the amplitude of the manoeuvre according to their sensation.

Forced vital capacity (FVC) and forced expiratory volume in $1 \mathrm{~s}\left(\mathrm{FEV}_{1}\right)$ were measured in the sitting position using a spirometer.

The DMD patient was 33 years old, with severe muscular weakness, severe dysphagia, using cough assisted device and continuous non-invasive ventilation. The patient was able to stay disconnected from the ventilator and autonomously breathe for short periods of time. During the periods disconnected from the ventilator, his spontaneous breathing was characterised by 3-4 tidal breaths alternating with GPB. Spirometry was unavailable in this patient due to the impossibility of obtaining a reliable test because of the severity of the disease, namely facial muscle weakness causing an impediment to holding the mouthpiece with no leakage. He performed the latest spirometry in 2009 (FVC $0.4 \mathrm{~L}, 8 \%$ pred; FEV ${ }_{1}$ not available). The BMD patient was 19 years old, ambulant, FVC $2.62 \mathrm{~L}$ (55\% pred), FEV $12.10 \mathrm{~L}$ (51\% pred), peak cough flow $287 \mathrm{~L} \cdot \mathrm{min}^{-1}$, not using any respiratory assistive device. The HC was a 29 -year-old male competitive breath-hold diver, FVC $6.40 \mathrm{~L}$ (106\% pred) and $\mathrm{FEV}_{1} 4.98 \mathrm{~L}$ (107\% pred).

All subjects signed a written informed consent form approved by the local ethical committee according to the declaration of Helsinki. The DMD patient also signed an informed consent for the publication of his video in the supplementary material associated to this research letter.

Because of his age, the specific form of the dystrophy and its progression, the DMD patient was characterised by a severe ventilatory and restrictive pattern. In the DMD and BMD patients, as well as in $\mathrm{HC}$, respectively, the following data were measured in the supine position during quiet spontaneous breathing: respiratory rate 36,15 and 8 breaths $\mathrm{min}^{-1}$; tidal volume, 118,457 and $930 \mathrm{~mL}$ resulting in a rapid and shallow breathing index (RSBI) of 317,32 and 9 breaths $\cdot \min ^{-1} \cdot \mathrm{L}^{-1}$ (figure 1). RSBI is defined as the ratio of respiratory frequency to tidal volume and it is an important parameter used to predict successful weaning of mechanical ventilation on intensive care units. A respiratory rate of $>38$ breaths $\cdot \mathrm{min}^{-1}$ and an RSBI of $>100$ breaths $\cdot \mathrm{min}^{-1} \cdot \mathrm{L}^{-1}$ appear to reduce the probability of successful extubation [14].

Respiratory rate (DMD 29; BMD 17; HC 6 breaths. $\min ^{-1}$ ), tidal volume (DMD 150; BMD 618; HC $1285 \mathrm{~mL}$ ) and RSBI (DMD 208; BMD 30; HC 5 breaths $\cdot \mathrm{min}^{-1} \cdot \mathrm{L}^{-1}$ ) were also measured in the seated position.

In supine position, IC was $0.25,1.9$ and $4.7 \mathrm{~L}$, whereas in seated position it was $0.21,1.77$ and $4.3 \mathrm{~L}$, respectively, in the DMD and BMD patients and HC. As expected, at the end of the GPB period all the subjects expanded their chest wall well above the volume reached with the IC manoeuvre, the DMD and BMD patients and the $\mathrm{HC}$, respectively, reaching $2.2 \mathrm{~L}(\sim 880 \%$ of IC), $2.5 \mathrm{~L}(\sim 131 \%$ of IC) and $5.4 \mathrm{~L}$ ( $\sim 115 \%$ of IC). This was achieved by gulps averaging 45,40 and $74 \mathrm{~mL}$, respectively, in the DMD and BMD patients and HC when lying supine (figure 1). Similarly, in the seated position after maximal GPB the volumes reached were $\sim 495 \%, \sim 121 \%$ and $\sim 113 \%$ of IC, respectively in the DMD and BMD patients and $\mathrm{HC}$, with gulps of 36,55 and $77 \mathrm{~mL}$.

GPB has another important effect on abdominal expansion. While ribcage and abdominal volume variations were perfectly in phase in $\mathrm{BMD}$ and $\mathrm{HC}$ at rest and during IC, they were out of phase in the DMD patient in supine position. For this reason, the contribution of the abdomen in DMD was negative (inspiratory paradox) both at rest $(-14 \%)$ and during IC $(-13 \%)$, while it was positive in both the BMD patient (rest: 51\%; IC: 42\%) and HC (rest: 68\%, IC: 37\%). Similarly, in the seated position, inspiratory paradox of the abdominal compartment was still evident in the DMD patient, with a negative contribution both at rest $(-3.5 \%)$ and during IC $(-1.8 \%)$, whereas it was still positive in both the BMD patient (rest $22 \%$; IC 20\%) and HC (rest 57\%, IC 32\%).

During GPB ribcage and abdomen expanded in phase in all subjects (figure 1).

In health, during inspiration the diaphragm contracts, moving caudally with a piston-like movement that makes abdominal pressure and volume increase and pleural pressure become sub-atmospheric to generate inspiratory flow [15]. When the diaphragm is severely weak or paralysed, as in the DMD patient, the ribcage muscles lead inspiration [12]. Their contraction generates the negative pleural pressure gradient that is partially transmitted to the abdomen because the diaphragm is not able to counteract it. A paradoxical inward movement of the abdominal compartment therefore occurs. The presence of out of 

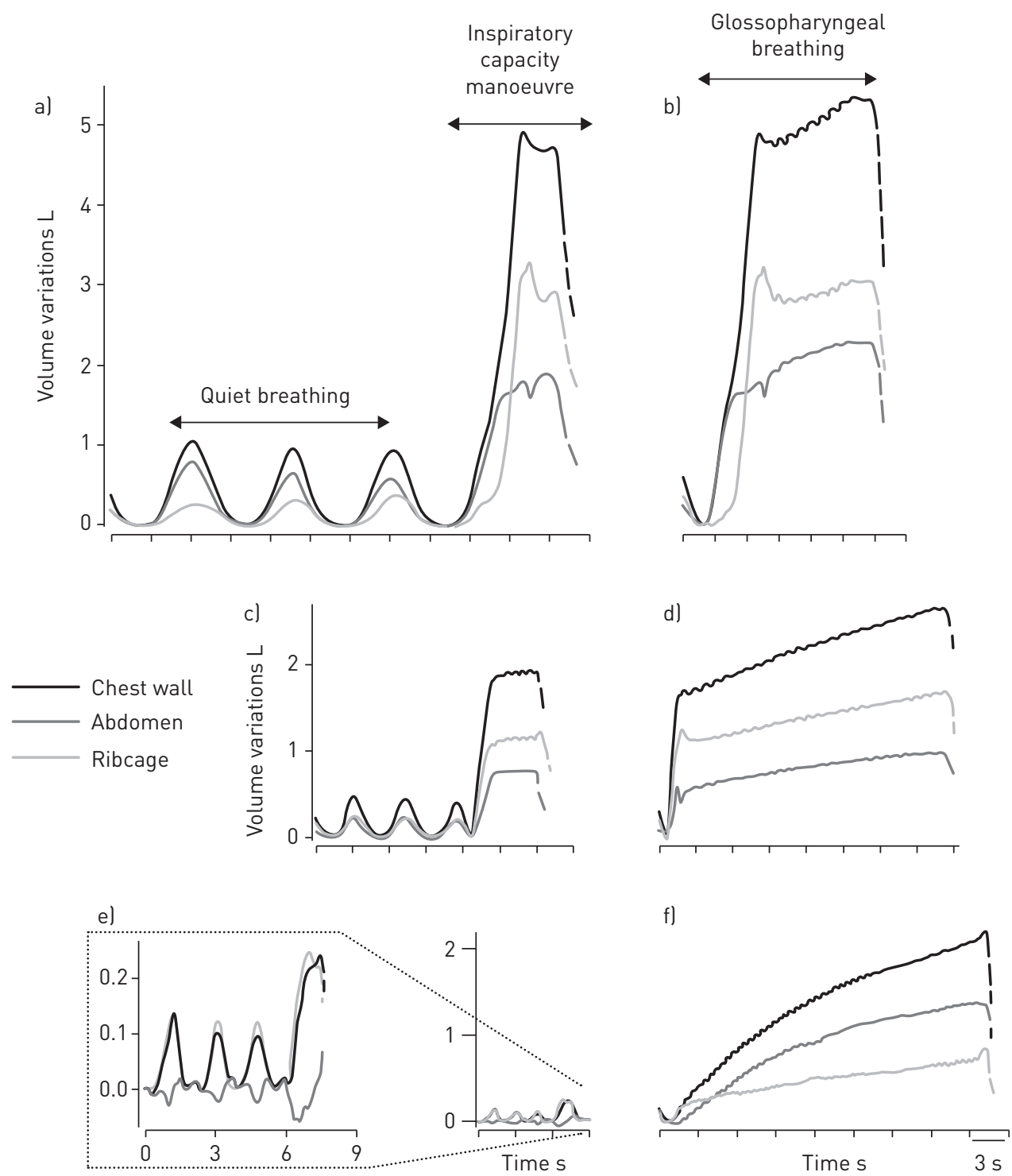

FIGURE 1 Volume variations of the ribcage, the abdomen and the chest wall during three breaths followed by an inspiratory capacity ( $a, c$ and e) and during glossopharyngeal breathing (b, $d$ and $f$ ) in a competitive breath-hold diver ( $a$ and b), and patients with Becker muscular dystrophy (c and d) and Duchenne muscular dystrophy (e and f).

phase thoraco-abdominal movement determines an inhomogeneous distribution of lung ventilation, with a reduction of ventilation in the lower parts of the lung due to the cranial shift of the diaphragm during inspiration. By contrast, GPB, driven by oropharyngeal muscles, represents a positive pressure breathing technique that allows to increase, presumably in a stepwise way, alveolar pressure homogeneously in the lung. The expansion of the lower part of the lung is transmitted to the abdomen, through a passive diaphragm, that finally expands.

We have shown how the volumes change during GPB in competitive breath-hold diver, in a mild and a severe MD patient and that GPB is effective also in presence of severe dysphagia and severe diaphragmatic weakness.

The net effect of GPB seemed milder in the healthy diver compared to MD patients, particularly the most severely compromised one. At the end of GPB, in fact, the increment of volume over IC was substantially greater in the MD patients than the diver. Although flow and pressure were not measured, it can be speculated that GPB mainly resulted in gas compression in $\mathrm{HC}$ and in volume increment in the MD patients. MD patients, although characterised by lung restriction due to the weak respiratory muscles, were able to recruit a substantial additional inspiratory volume by using glossopharyngeal muscles. 
The expansion was mainly thoracic in $\mathrm{HC}$ and the BMD patient during GPB, but confined to the abdomen in the DMD patient. This might be explained to be a consequence of the latter having a more rigid thorax, because of a reduced distensibility of the lung and the stiffer chest wall, while the abdomen became more compliant [9].

GPB not only expands the lung beyond IC, but it also reverses paradoxical respiratory abdominal motion with a consequent presumably more homogenous distribution of pulmonary ventilation (i.e. an increased expansion of the lung basis, reflected as a larger diaphragm displacement).

Our data from the DMD patient, in addition, confirmed that GPB is essential for allowing him to stay disconnected from the ventilator, even for short periods of time, and to assist phonation and cough (when cough-assisted device was not available) by modulating the amplitude of the inspiratory volume.

For all these reasons, our data suggest that patients with intact bulbar function, even in presence of muscular weakness/atrophy/paralysis, should be trained and/or encouraged to perform GPB daily.

Antonella LoMauro (ib ${ }^{1}$, Paolo Banfi ${ }^{2}$, Maria Grazia D’Angelo ${ }^{3}$ and Andrea Aliverti ${ }^{1}$

${ }^{1}$ Dipartimento di Elettronica, Informazione e Bioingegneria, Politecnico di Milano, Milan, Italy. ${ }^{2}$ IRCCS Fondazione Don Carlo Gnocchi, Milan, Italy. ${ }^{3}$ Scientific Institute IRCCS E. Medea, Neuromuscular Unit, Lecco, Italy.

Correspondence: Antonella LoMauro, Politecnico di Milano, Dipartimento di Elettronica, Informazione e Bioingegneria, piazza L. Da Vinci, Milan 20133, Italy. E-mail: antonella.lomauro@polimi.it

This article has supplementary material available from erj.ersjournals.com

Received: Oct 092018 | Accepted after revision: April 182019

Conflict of interest: None declared.

\section{References}

Collier CR, Dail CW, Affeldt JE. Mechanics of glossopharyngeal breathing. J Appl Physiol 1956; 8: 580-584.

Maltais F. Glossopharyngeal Breathing. Am J Respir Crit Care Med 2011; 184: 381-381.

Loring SH, O’Donnell CR, Butler JP, et al. Transpulmonary pressures and lung mechanics with glossopharyngeal insufflation and exsufflation beyond normal lung volumes in competitive breath-hold divers. J Appl Physiol 2007; 102: 841-846.

4 Boussuges A, Gavarry O, Bessereau J, et al. Glossopharyngeal insufflation and breath-hold diving: the more, the worse? Wilderness Environ Med 2014; 25: 466-471.

5 Bach JR, Bianchi C, Vidigal-Lopes M, et al. Lung inflation by glossopharyngeal breathing and "air stacking" in Duchenne muscular dystrophy. Am J Phys Med Rehabil 2007; 86: 295-300.

6 Seccombe LM, Chung SCS, Jenkins CR, et al. Lung perfusion and chest wall configuration is altered by glossopharyngeal breathing. Eur Respir J 2010; 36: 151-156.

7 Lindholm P, Lundgren CE. The physiology and pathophysiology of human breath-hold diving. J Appl Physiol 2009; 106: 284-292.

8 Nygren-Bonnier M, Schiffer TA, Lindholm P. Acute effects of glossopharyngeal insufflation in people with cervical spinal cord injury. J Spinal Cord Med 2018; 41: 85-90.

9 LoMauro A, Aliverti A. Physiology of respiratory disturbances in muscular dystrophies. Breathe 2016; 12 : 318-327.

10 Bianchi C, Carrara R, Khirani S, et al. Independent cough flow augmentation by glossopharyngeal breathing plus table thrust in muscular dystrophy. Am J Phys Med Rehabil 2014; 93: 43-48.

11 Aliverti A, Dellacà R, Pelosi P, et al. Compartmental analysis of breathing in the supine and prone positions by optoelectronic plethysmography. Ann Biomed Eng 2001; 29: 60-70.

12 Romei M, Mauro AL, D’Angelo MG, et al. Effects of gender and posture on thoraco-abdominal kinematics during quiet breathing in healthy adults. Respir Physiol Neurobiol 2010; 172: 184-191.

13 LoMauro A, Romei M, Gandossini S, et al. Evolution of respiratory function in Duchenne muscular dystrophy from childhood to adulthood. Eur Respir J 2018; 51: 1701418.

14 Meade M, Guyatt G, Cook D, et al. Predicting success in weaning from mechanical ventilation. Chest 2001; 120: 400S-424S.

15 Macklem PT. Respiratory muscles: the vital pump. Chest 1980; 78: 753-758. 